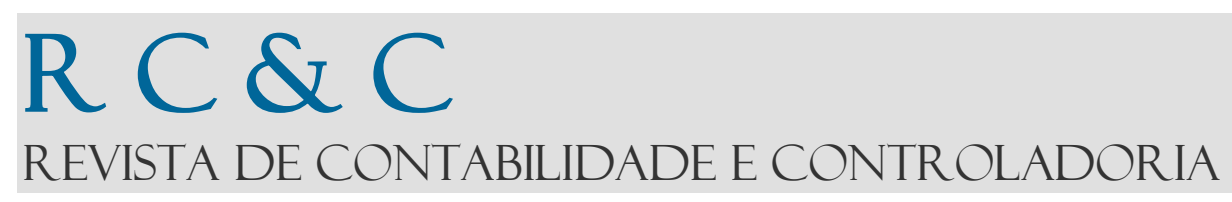

\title{
ÍNDICE POR TÍTULOS 2012
}

A ANÁLISE COMPORTAMENTAL DOS ATIVOS INTANGÍVEIS: UM ESTUDO NAS COMPANHIAS DA BM\&FBOVESPA.

Jéssica de Oliveira Rech; Darci Schnorrenberger; Rogério João Lunkes

Curitiba, v. 4, n.2, p. 52-68, maio/ago. 2012

ANÁLISE COMPARATIVA DO DESEMPENHO CONTÁBIL-FINANCEIRO DE EMPRESAS COM BOAS PRÁTICAS DE GOVERNANÇA CORPORATIVA NO BRASIL

Marcelo Alvaro da Silva Macedo; Luiz João Corrar

Curitiba, v. 4, n.1, p 42-61, jan./abr. 2012

ANÁLISE DO ALINHAMENTO ENTRE AS PRÁTICAS DE GOVERNANÇA CORPORATIVA EVIDENCIADAS NOS RELATÓRIOS DA ADMINISTRAÇÃO: UM ESTUDO NAS EMPRESAS DO NÍVEL NOVO MERCADO DA BM\&FBOVESPA

Deisy Cristina Corrêa Igarashi; Reinaldo Rodrigues Camacho; Wagner Igarashi; Silvane Zandoná

Curitiba, v. 4, n.2, p.90-109, maio/ago. 2012

ANÁLISE DO NÍVEL DE CONSERVADORISMO CONDICIONAL DAS EMPRESAS BRASILEIRAS LISTADAS NA BM\&FBOVESPA APÓS A ADOÇÃO DOS IFRS.

Roselaine Filipin; Silvio Aparecido Teixeira; Francisco Antonio Bezerra; Paulo Roberto da Cunha

Curitiba, v. 4, n.2, p. 24-36, maio/ago. 2012

ANÁLISE DOS FATORES DETERMINANTES DA ESTRUTURA DE CAPITAL DE COMPANHIAS DE CONSTRUÇÃO CIVIL INSERIDAS NO SEGMENTO BOVESPA

Danillo Lemes Gonçalves; Oscar Neto de Almeida Bispo

Curitiba, v. 4, n.1, p 110-130, jan./abr. 2012

ANALYSIS OF THE RESIDUAL INCOME VALUATION AND ABNORMAL EARNINGS GROWTH MODELS: A PRACTICAL APPROACH USING ANALYSTS' FORECASTS

José Elias Feres de Almeida; Gerlando Augusto Sampaio Franco de Lima; Iran Siqueira Lima; José Roberto Securato

Curitiba, v. 4, n.1, p 7-19, jan./abr. 2012

ATRIBUTOS DE UM BOM PROFESSOR: UM ESTUDO SOBRE A PERCEPÇÃO DOS ALUNOS DE CIÊNCIAS CONTÁBEIS

Vagner Antonio Marques; Marleide Cerqueira de Oliveria;Eduardo Mendes Nascimento; Jacqueline Veneroso Alves da Cunha

Curitiba, v. 4, n.2, p. 7-23, maio/ago. 2012

BALANCED SCORECARD: UM ESTUDO BIBLIOMÉTRICO ACERCA DA PRODUÇÃO ACADÊMICA DA DÉCADA DE 2001-2011 NO BRASIL

Florindo Rhaoni Picoli; Gustavo Abib; Marcos Wagner Da Fonseca

Curitiba, v. 4, n.3, p. 128-142, set./dez. 2012

Revista de Contabilidade e Controladoria, ISSN 1984-6266

Universidade Federal do Paraná, Curitiba, v. 4, n.3, p 154-156, set./dez. 2012. 
CONSERVADORISMO CONTÁBIL: UMA ANÁLISE DOS RESULTADOS DAS EMPRESAS LISTADAS NA BOLSA DE VALORES CHINESAS

Jomar Miranda Rodrigues; Jorge Katsumi Niyama; Pedro Henrique Duarte Oliveira

Curitiba, v. 4, n.1, p 93-109, jan./abr. 2012

CONTABILIDADE E INSTITUCIONALISMO ORGANIZACIONAL :FUNDAMENTOS E IMPLICAÇÕES PARA A PESQUISA

Edson Ronaldo Guarido Filho; Mayla Cristina Costa

Curitiba, v. 4, n.1, p 20-41, jan./abr. 2012

DEMONSTRAÇÃO DOS FLUXOS DE CAIXA: ANÁLISE DE DIFERENÇAS DE PROCEDIMENTOS DE DIVULGAÇÃO ENTRE EMPRESAS LISTADAS NAS BOLSAS DE VALORES DE SÃO PAULO, FRANKFURT, MILÃO E LONDRES

Luciano Márcio Scherer; Jocelino Donizetti Teodoro; Sonia Raifur Kos; Raquel Prediger Anjos

Curitiba, v. 4, n.2, p. 37-51, maio/ago. 2012

DETERMINANTES DE CUSTOS EM EMPRESAS DE PEQUENO PORTE DO SETOR MOVELEIRO: UM ESTUDO EM EMPRESAS DA SERRA GAÚCHA

Marcos Antonio de Souza; Franciele Mezzomo

Curitiba, v. 4, n.1, p 131-149, jan./abr. 2012

ESTÁGIO ATUAL DE EVIDENCIAÇÃO E FATORES QUE INFLUENCIAM O DISCLOSURE DE INFORMAÇÕES POR SEGMENTOS POR EMPRESAS BRASILEIRAS

Flávia de Araújo e Silva; Laura Edith Taboada Pinheiro

Curitiba, v. 4, n.3, p. 78-94, set./dez. 2012

ESTUDO SOBRE AS DIFERENÇAS DE DESEMPENHO ENTRE PEQUENAS E MÉDIAS EMPRESAS BRASILEIRAS: UMA ABORDAGEM DA VISÃO BASEADA EM RECURSOS (VBR)

Geraldo Alemandro Leite Filho; Francisval de Melo Carvalho; Luiz Marcelo Antonialli

Curitiba, v. 4, n.1, p 78-92, jan./abr. 2012

GERENCIAMENTO DE RESULTADOS EM EMPRESAS LATINO-AMERICANAS COM ADR'S NEGOCIADOS NOS ESTADOS UNIDOS

Régis Augusto Sandrin; Rodrigo Oliveira Soares

Curitiba, v. 4, n.3, p. 113-127, set./dez. 2012

IMPACTOS DE MESTRADOS ESPECIAIS EM CONTABILIDADE NA TRAJETÓRIA DE SEUS EGRESSOS:

UM OLHAR ESPECIAL PARA GÊNERO

Silvia Pereira de Castro Casa Nova

Curitiba, v. 4, n.3, p. 37-62, set./dez. 2012

Revista de Contabilidade e Controladoria, ISSN 1984-6266

Universidade Federal do Paraná, Curitiba, v. 4, n.3, p 154-156, set./dez. 2012. 
ÍNDICE DE SUSTENTABILIDADE EMPRESARIAL (ISE) E DESEMPENHO FINANCEIRO DAS EMPRESAS DO SETOR DE PAPEL E CELULOSE

Emanuelle Cristine de Melo; Fernanda Maria de Almeida; Gislaine Aparecida da Silva Santana

Curitiba, v. 4, n.3, p. 95-112, set./dez. 2012

JOGOS DE EMPRESAS NA RELAÇÃO ENSINO-APRENDIZAGEM:

UMA AVALIAÇÃO NA FORMAÇÃO DO BACHAREL EM CIÊNCIAS CONTÁBEIS

Ruth Gonçalves Duarte; Diego de Queiroz Machado;

Fátima Regina Ney Matos; Maria Clara Cavalcante Bugarim; Diana Macedo Matos

Curitiba, v. 4, n.3, p. 63-77, set./dez. 2012

NORMAS CONTÁBEIS DE RECONHECIMENTO E MENSURAÇÃO DO ATIVO IMOBILIZADO:

IMPACTO EM EMPRESAS DE BENS INDUSTRIAIS GAÚCHAS LISTADAS NA BM\&FBOVESPA

Alex Eckert; Marlei Salete Mecca; Roberto Biasio; Karmine Mattiuz de Souza

Curitiba, v. 4, n.2, p.69-89, maio/ago. 2012

O TABLEAU DE BORD E O BALANCED SCORECARD: UMA ANÁLISE COMPARATIVA

Patrícia Rodrigues Quesado; Beatriz Aibar Guzmán; Lúcia Lima Rodrigues

Curitiba, v. 4, n.2, p.128-150, maio/ago. 2012

PLANEJAMENTO TRIBUTÁRIO E AS PRÁTICAS DE RESPONSABILIDADE SOCIAL CORPORATIVA

Beatriz Santos Neves Fadlalah; Antonio Lopo Martinez; Valcemiro Nossa

Curitiba, v. 4, n.3, p. 7-23, set./dez. 2012

REFLEXOS DAS ALTERAÇÕES DA LEI № 11.638/07 NAS INFORMAÇÕES TRIMESTRAIS DE EMPRESAS DE CAPITAL ABERTO

Julio Orestes da Silva; Darclê Costa Silva Haussmann; Ilse Maria Beuren

Curitiba, v. 4, n.1, p 62-77, jan./abr. 2012

UM ESTUDO MULTICASO SOBRE A GESTÃO DE RECURSOS FINANCEIROS EM ORGANIZAÇÕES DO TERCEIRO SETOR

Leandro da Costa Lopes; Cacilda Soares de Andrade; Juliana Matos de Meira; Aldemar Araújo dos Santos; Joaquim Osório Liberalquino Ferreira

Curitiba, v. 4, n.3, p. 24-36, set./dez. 2012

VALOR JUSTO APLICADO NOS INSTRUMENTOS FINANCEIROS: UM ESTUDO NAS PESQUISAS NACIONAIS E INTERNACIONAIS

Tatiane de Oliveira Marques; Charles Albino Schultz; Gertrudes Aparecida Dandolini; João Artur de Souza; Sergio Murilo Petri

Curitiba, v. 4, n.2, p 110-127, maio/ago. 2012

Revista de Contabilidade e Controladoria, ISSN 1984-6266

Universidade Federal do Paraná, Curitiba, v. 4, n.3, p 154-156, set./dez. 2012. 